\title{
Accuracy of Visual Inspection with Acetic acid in Detecting High-Grade Cervical Intraepithelial Neoplasia in Pre- and Post-Menopausal Thai Women with Minor Cervical Cytological Abnormalities
}

\author{
Yenrudee Poomtavorn*, Komsun Suwannarurk
}

\begin{abstract}
Purpose: To determine the accuracy of visual inspection with acetic acid (VIA) in detecting high-grade cervical intraepithelial neoplasia (CIN) in pre- and post-menopausal women with atypical squamous cells of undetermined significance (ASC-US) and low grade squamous intraepithelial lesion (LSIL) Papanicolaou (Pap) smears. Materials and Methods: Two hundred women (150 pre-menopausal and 50 post-menopausal) with ASCUS and LSIL cytology who attended the colposcopy clinic, Thammasat University Hospital, between March 2013 and August 2014 were included. All women underwent VIA testing and colposcopy by gynecologic oncologists. Diagnostic values of VIA testing including sensitivity, specificity, positive predictive value (PPV) and negative predictive value (NPV) for detecting high-grade CIN were determined using the histopathology obtained from colposcopic-directed biopsy as a gold standard. Results: VIA testing was positive in 54/150 (36\%) pre-menopausal women and 5/50 (10\%) post-menopausal women. Out of 54 pre-menopausal women with positive VIA testing, $15(27.8 \%)$ had high-grade CIN and $39(72.2 \%)$ had either CIN 1 or insignificant pathology. Ten (10.4\%), 43 $(44.8 \%)$ and $43(44.8 \%)$ out of the remaining 96 pre-menopausal women with negative VIA testing had highgrade CIN, CIN 1 and insignificant pathology, respectively. Out of 5 post-menopausal women with positive VIA testing, there were $4(80 \%)$ women with high-grade CIN, and $1(20 \%)$ women with insignificant pathology. Out of 45 VIA-negative post-menopausal women, $42(93.3 \%)$ women had CIN 1 and insignificant pathology, and 3 (6.7\%) had high-grade CIN. Sensitivity, specificity, PPV and NPV of the VIA testing were 59.4\%, 76.2\%, 32.2\% and $90.8 \%$, respectively $(60 \%, 68.8 \%, 27.8 \%$ and $89.6 \%$ in pre-menopausal women and $57.1 \%, 97.7 \%, 80 \%$ and $93.3 \%$ in post-menopausal women). Conclusions: VIA testing may be used as a screening tool for detecting high-grade CIN in women with minor cervical cytological abnormalities in a low-resource setting in order to lower the rate of colposcopy referral.
\end{abstract}

Keywords: Visual inspection with acetic acid - high-grade CIN - Papanicolaou smear - Thailand

Asian Pac J Cancer Prev, 16 (6), 2327-2331

\section{Introduction}

Cervical cytologic screening by Papanicolaou (Pap) smear is a standard method for cervical cancer screening. The Bethesda system 2001 has categorized the abnormal cervical cytology of squamous epithelium into atypical squamous cells (ASC), low grade squamous intraepithelial lesion (LSIL), high grade squamous intraepithelial lesion (HSIL), and squamous cell carcinoma (SCC) (Solomon et al., 2002). ASC and LSIL generally have underlying low grade abnormal histopathology including inflammation, cervical intraepithelial neoplasia (CIN) 1 and human papillomavirus (HPV) infection which may spontaneously regress. Unlike ASC and LSIL, HSIL and SCC usually represent high-grade lesions including CIN 2, CIN 3 and invasive cancer requiring definite investigation and management. By using colposcopic-directed biopsy, the incidences of CIN 2-3 in patients with atypical squamous cells of undetermined significance (ASC-US) and LSIL ranged from $7-15 \%$ and $15-30 \%$, respectively and the incidences of cervical cancer in patients with ASC-US and LSIL were reported at $0.1-2 \%$ and $1-5 \%$, respectively (Chute et al., 2006; Evans et al., 2006; Kantathavorn et al., 2008; Khuakoonratt et al., 2008).

The options for the management of women with ASC-US cytology include reflex HPV testing and repeat the cervical cytology at 1 year following the ASC-US cytology diagnosis (Massad et al., 2013). Colposcopy is recommended for women with HPV-positive ASC-US from either reflex HPV testing or cotesting and for LSIL cytology-women with either no HPV result or positive high-risk HPV (HR-HPV) (Massad et al., 2013). In 
Yenrudee Poomtavorn and Komsun Suwannarurk

women with LSIL cytology with negative HR-HPV, repeat cotesting at 1 year following the LSIL cytology diagnosis is preferred, but colposcopy is acceptable (Massad et al., 2013). However, HPV testing may not be available in a low-resource setting including some areas of Thailand owing to its high cost and sophisticated laboratories requirement. In addition, a follow-up cervical cytology potentially possesses some disadvantages such as anxiety of patients, loss of follow-up visits of patients and delay in diagnosis and treatment of occult high-grade CIN. Furthermore, colposcopy requires well-trained colposcopists. Because of these limitations, it is therefore necessary to have a cost-effective method to identify the underlying high-grade CIN in women with minor cervical cytological abnormalities in a low-resource setting in order to promptly diagnose and treat it.

Visual inspection with acetic acid (VIA) testing is a safe and inexpensive cervical cancer screening method that provides an immediate result and treatment. It requires more simple instruments as compared with those required by cervical cytology, HPV testing and colposcopy. In addition, VIA testing can be performed by trained paramedical personnel and it has a relatively high sensitivity (Bradford and Goodman, 2013). A recent mataanalysis reported sensitivity, specificity, PPV and NPV of VIA testing in detecting high-grade CIN in asymptomatic women by using CIN 2 as the disease threshold at $80 \%, 92 \%, 10 \%$ and $99 \%$, respectively (Sauvaget et al., 2011).

The primary objective of this study was to evaluate the accuracy of VIA testing in detecting high-grade CIN in women with ASC-US and LSIL cytology. The secondary objective was to compare the accuracy of VIA testing in detecting high-grade $\mathrm{CIN}$ in pre- and post-menopausal women.

\section{Materials and Methods}

Women, aged 21-65 years, with ASC-US and LSIL cytology who attended the colposcopy clinic, Thammasat University Hospital between March 2013 and August 2014 were enrolled. Women who had a history of precancerous lesion of cervix and invasive cervical cancer, pregnancy and who had undergone hysterectomy were excluded. The study was approved by the Ethics Committee of Thammasat University. Informed consent was obtained from all participants.

Clinical data including age, parity, menopausal status and cervical cytology were collected. VIA testing and colposcopic-directed biopsy and endocervical sampling (ECS) were undertaken. The first gynecologic oncologist (K.S.) performed VIA testing by naked eye inspection of the cervix at one minute after the application of $3 \%$ acetic acid over the cervix. The VIA testing results were categorized into either negative or positive tests for cervical neoplasia. A positive test was characterized by well-defined, opaque acetowhite lesions in the transformation zone closed to squamo-columnar junction (SCJ) (Sankaranarayanan and Wesley, 2003). A negative test was characterized by one or more of the following lesions: no acetowhite lesions, ill-defined, faint, translucent acetowhite lesions, acetowhitening of endocervical polyps, nabothian cysts, prominent acetowhitening of the SCJ, dot-like acetowhitening scattered all over the cervix and geographic satellite acetowhite lesions not touching the SCJ (Sankaranarayanan and Wesley, 2003). After the VIA testing, the colposcopic examination and colposcopic-directed biopsy was performed by the second gynecologic oncologist (Y.P.) who was blinded to the VIA testing result. Appropriate management was provided for patients according to the pathological results of colposcopic-directed biopsy and ECS.

Sample size was calculated based on the prevalence of high-grade CIN in women with ASC-US and LSIL cytology of $15 \%$ and $95 \%$ sensitivity of VIA testing to detect high-grade CIN from the previous reports (Evans et al., 2006; Aggarwal et al., 2011; Hasanzadeh et al., 2011). The calculated sample size was 200. Data were analyzed using SPSS version 15.0. Descriptive statistics were used for demographic data. Chi-square test was used to evaluate the difference between the categorical variables. Accuracy of the VIA testing including sensitivity, specificity, PPV and NPV were determined using the histopathology result of colposcopic-directed biopsy and/or ECS as a gold standard.

\section{Results}

Two hundred women (150 pre-menopausal and 50 post-menopausal) with ASC-US and LSIL cytology were enrolled in the study. Mean \pm SD age of all enrolled women was $40 \pm 11$ years $(35 \pm 8$ and $53 \pm 6$ years for pre- and postmenopausal women, respectively). Majority of the enrolled women were multiparous (Table 1). There were 80 out of $150(53.3 \%)$ and 70 out of $150(46.7 \%)$ pre-menopausal women with ASC-US and LSIL cytology, respectively. While 38 out of $50(76 \%)$ post-menopausal women had ASC-US and the remaining $12(24 \%)$ post-menopausal women had LSIL cytology. The VIA testing was positive in 59 out of $200(29.5 \%)$ patients enrolled (54 (36\%) and $5(10 \%)$ of pre-menopausal and post-menopausal women, respectively). The histopathology results of the colposcopic-directed biopsy are shown in Table 1. CIN 2-3 were demonstrated in $25(16.7 \%)$ pre-menopausal women and 7 (14\%) post-menopausal women. CIN 1-3 were present in ECS tissues of $10(6.7 \%)$ and 10 (20\%) pre- and post-menopausal women, respectively.

CIN 2-3 were demonstrated in 12 out of $80(15 \%)$ and 13 out of $70(18.6 \%)$ pre-menopausal women with ASC-US and LSIL cytology, respectively. Seven out of 38 (18.4\%) post-menopausal women with ASC-US cytology had CIN 2-3, while no post-menopausal women with LSIL cytology had CIN 2-3 (Table 2).

Table 3 shows histopathology results of the colposcopicdirected biopsy according to the VIA testing results. Out of 54 pre-menopausal women with positive VIA testing, $15(27.8 \%)$ had high-grade CIN and $39(72.2 \%)$ had either CIN 1 or insignificant pathology. Ten (10.4\%), 43 (44.8\%) and $43(44.8 \%)$ out of the remaining 96 pre-menopausal women with negative VIA testing had high-grade CIN, CIN 1 and insignificant pathology, respectively. Out of 5 post-menopausal women with positive VIA testing, there 
Table 1. Clinical Characteristics of All 200 Enrolled Patients

\begin{tabular}{lcc}
\hline Characteristics & $\begin{array}{c}\text { Pre-menopausal, } \\
(\mathrm{N}=150)\end{array}$ & $\begin{array}{c}\text { Post-menopausal, } \\
\mathrm{N}=50) \\
\mathrm{N}(\%)\end{array}$ \\
\hline Parity $(\%)$ \\
$\quad$ Nulliparous & $51(34)$ & $3(6)$ \\
Multiparous & $99(66)$ & $47(94)$ \\
Pap smear results & & \\
ASC-US & $80(53.3)$ & $38(76.0)$ \\
LSIL & $70(46.7)$ & $12(24.0)$ \\
VIA testing & & \\
Positive & $54(36)$ & $5(10)$ \\
Negative & $96(64)$ & $45(90)$ \\
Colposcopy & & \\
Satisfactory & $88(58.7)$ & $17(34.0)$ \\
Unsatisfactory & $62(41.3)$ & $33(66.0)$ \\
Pathology of colposcopic-directed biopsy & \\
No CIN & $61(40.6)$ & $29(58.0)$ \\
CIN 1 & $64(42.7)$ & $14(28.0)$ \\
CIN 2-3 & $25(16.7)$ & $7(14)$ \\
Pathology of ECS & & \\
No CIN & $140(93.3)$ & $40(80.0)$ \\
CIN 1-3 & $10(6.7)$ & $10(20.0)$ \\
\hline
\end{tabular}

*ASC-US, atypical squamous cells of undetermined significance; LSIL, low grade squamous intraepithelial lesion; CIN, cervical intraepithelial neoplasia; VIA, visual inspection with acetic acid; ECS, endocervical sampling

Table 2. Histopathology Results of Colposcopicdirected Biopsy According to the Cervical Cytology Results

\begin{tabular}{llll}
\hline Cervical cytology & \multicolumn{3}{c}{ Colposcopic-directed biopsy pathology, } \\
\cline { 2 - 4 } & No CIN & CIN 1 & CIN 2-3 \\
\hline Pre-menopausal (N=150) & & & \\
ASC-US (N=80) & $36(45.0)$ & $32(40.0)$ & $12(15.0)$ \\
LSIL (N=70) & $25(35.7)$ & $32(45.7)$ & $13(18.6)$ \\
Post-menopausal (N=50) & & & \\
ASC-US (N=38) & $17(44.7)$ & $14(36.8)$ & $7(18.4)$ \\
LSIL (N=12) & $12(100)$ & $0(0)$ & $0(0)$ \\
\hline
\end{tabular}

*ASC-US, atypical squamous cells of undetermined significance; LSIL, low grade squamous intraepithelial lesion; CIN, cervical intraepithelial neoplasia

Table 3. Histopathology Results of the ColposcopicDirected Biopsy According to the VIA Results

\begin{tabular}{llll}
\hline \multicolumn{4}{c}{ Colposcopic-directed biopsy pathology, } \\
\cline { 2 - 4 } VIA results & \multicolumn{3}{c}{$\mathrm{N}(\%)$} \\
\hline Pre-menopausal $(\mathrm{N}=150)$ & & \multicolumn{1}{c}{ CIN 1 } & CIN 2-3 \\
VIA positive $(\mathrm{N}=54)$ & $18(33.3)$ & $21(38.9)$ & $15(27.8)$ \\
VIA negative $(\mathrm{N}=96)$ & $43(44.8)$ & $43(44.8)$ & $10(10.4)$ \\
Post-menopausal $(\mathrm{N}=50)$ & & & \\
VIA positive $(\mathrm{N}=5)$ & $1(20)$ & $0(0)$ & $4(80)$ \\
VIA negative $(\mathrm{N}=45)$ & $28(62.2)$ & $14(31.1)$ & $3(6.7)$ \\
\hline
\end{tabular}

*CIN, cervical intraepithelial neoplasia; VIA, visual inspection with acetic acid

were 4 (80\%) women with high-grade CIN, and 1 (20\%) women with insignificant pathology. Out of 45 VIAnegative post-menopausal women, $42(93.3 \%)$ women had CIN 1 and insignificant pathology, and $3(6.7 \%)$ had high-grade CIN.
Pre-menopausal women with positive VIA testing who had higher degree of abnormal cytology (LSIL) had more frequency of having high-grade CIN (35.7\% for women with LSIL and $19.2 \%$ for women with ASC-US). All postmenopausal women with LSIL cytology had negative VIA testing and none of them had high-grade CIN.

Sensitivity, specificity, PPV and NPV of the VIA testing were $59.4 \%, 76.2 \%, 32.2 \%$ and $90.8 \%$, respectively $(60 \%$, $68.8 \%, 27.8 \%$ and $89.6 \%$ in pre-menopausal women and $57.1 \%, 97.7 \%, 80 \%$ and $93.3 \%$ in post-menopausal women).

\section{Discussion}

The present study demonstrated a relatively high rate of high-grade CIN in both pre- and post-menopausal women with ASC-US cytology (15.0\% vs $18.4 \%$, respectively). Limpvanuspong et al (Limpvanuspong et al., 2008) reported high-grade CIN in $9.1 \%$ of women with ASC-US cytology. The other study reported even lower rate of high-grade CIN in both pre- and post-menopausal women with ASC-US cytology at approximately 6\% (Goksedef et al., 2011). The rate of high-grade CIN in pre-menopausal women with LSIL in our study was concordant to the previous report by Khuakoonratt et al (Khuakoonratt et al., 2008) (18.6\% vs 15.0\%). However, none of post-menopausal women with LSIL cytology had high-grade CIN.

The present study reported positive VIA testing in $29.5 \%$ of the entire studied population which was higher than that reported in the previous studies (7-12\%) (Murillo et al., 2010; Phongsavan et al., 2011; Nessa et al., 2013; Castle et al., 2014). The discrepancy between the rate of positive VIA testing found in our study and the others could be explained by VIA testing performance in different groups of patients. Our study studied in women with abnormal cervical cytologic screening (ASC-US and LSIL cytology) while the previous studies performed VIA testing in asymptomatic normal population as a primary screening test. One of the important determinants for positive VIA testing is clinical symptoms of patients (symptomatic or asymptomatic). Cervical infection or inflammation, and area of transformation zone which is affected by age, menopausal status, parity and route of delivery have been shown to affect the result of VIA testing (Vedantham et al., 2010; Cremer et al., 2011; Castle et al., 2014).

The sensitivity of VIA testing in detecting highgrade CIN and cervical cancer ranged from $67-79 \%$ and the specificity varied from $49-86 \%$ in previous crosssectional studies which used VIA testing as a primary screening test (Denny et al., 2002; Cronje et al., 2003; Sankaranarayanan et al., 2004; Sankaranarayanan et al., 2005). Our study demonstrated overall sensitivity and specificity of $59.4 \%$ and $76.2 \%$, respectively of VIA testing in detecting high-grade CIN in women with minor cervical cytological abnormalities. The specificity was high at $97.7 \%$ in our studied patients who were post-menopausal. The strength of our study was that all women enrolled in the study had the VIA testing performed by a single gynecologic oncologist and the 
colposcopic examination also performed by a single different gynecologic oncologist who was blinded to the VIA testing results. These performance reduced the bias of the study. However, owing to the subjective nature of VIA testing and no standard criteria for positivity and negativity of VIA testing results, the accuracy of the VIA testing may be discrepant among the studies. In addition, experience of the personel who performed the VIA testing has an impact on the accuracy of VIA testing (Parashari and Singh, 2013).

However, another previous study showed no difference in the sensitivity and specificity of VIA testing performed in older women as compared with those performed in younger women (Sankaranarayanan et al., 2004; Sankaranarayanan et al., 2005; Dasgupta and Bhattacharya, 2012). In the elderly, regression of the transformation zone into the endocervix may diminish the adequacy and the sensitivity of VIA testing in detecting cervical dysplasia (Rochelson and Krumholz, 1983; Cremer et al., 2011). A previous study showed that the specificity of VIA testing was higher in post-menopausal women which is in agreement with our study (Denny et al., 2002). In addition, our study demonstrated better specificity, PPV and NPV of VIA testing in post-menopausal women than those of pre-menopausal women. However, the limitation was the small number of post-menopausal women included in this study and the low rate of positive CIN2-3 found on colposcopic-directed biopsy in this subgroup. Further larger studies of post-menopausal women are needed to confirm these results.

In conclusion, VIA testing may be used as a screening tool in detecting high-grade CIN in both pre- and postmenopausal women with minor cervical cytological abnormalities in low-resource settings where colposcopy is not widely available. Women with positive VIA testing should further undergo the colposcopy and women with negative VIA testing may undergo a follow-up cervical cytology.

\section{References}

Aggarwal P, Batra S, Gandhi G, Zutshi V (2011). Can visual inspection with acetic acid under magnification substitute colposcopy in detecting cervical intraepithelial neoplasia in low-resource settings? Arch Gynecol Obstet, 284, 397-403.

Bradford L, Goodman A (2013). Cervical cancer screening and prevention in low-resource settings. Clin Obstet Gynecol, 56, 76-87.

Castle PE, Qiao YL, Zhao FH, et al (2014). Clinical determinants of a positive visual inspection after treatment with acetic acid for cervical cancer screening. BJOG, 121, 739-46.

Chute DJ, Covell J, Pambuccian SE, Stelow EB (2006). Cytologic-histologic correlation of screening and diagnostic papanicolaou tests. Diagn Cytopathol, 34, 503-6.

Cremer M, Conlisk E, Maza M, et al (2011). Adequacy of visual inspection with acetic acid in women of advancing age. Int J Gynaecol Obstet, 113, 68-71.

Cronje HS, Parham GP, Cooreman BF, et al (2003). A comparison of four screening methods for cervical neoplasia in a developing country. Am J Obstet Gynecol, 188, 395-400.

Dasgupta S, Bhattacharya S (2012). Is visual inspection with acetic acid better than cervical cytology to screen women $>40$ years of age for carcinoma cervix? A cross-sectional study on proportion of screen-positive women (by VIA and cervical cytology) having CIN II/III lesion on cervical biopsy: difference between two age groups and among screening methods. Arch Gynecol Obstet, 285, 1731-6.

Denny L, Kuhn L, Pollack A, Wright TC Jr (2002). Direct visual inspection for cervical cancer screening: an analysis of factors influencing test performance. Cancer, 94, 1699-707.

Evans MF, Adamson CS, Papillo JL, et al (2006). Distribution of human papillomavirus types in thinprep papanicolaou tests classified according to the bethesda 2001 terminology and correlations with patient age and biopsy outcomes. Cancer, 106, 1054-64.

Goksedef BP, Akbayir O, Baran SY, et al (2011). Atypical squamous cells of undetermined significance in postmenopausal women: a comparative retrospective analysis. Eur J Obstet Gynecol Reprod Biol, 159, 418-21.

Hasanzadeh M, Esmaeili H, Tabaee S, Samadi F (2011). Evaluation of visual inspection with acetic acid as a feasible screening test for cervical neoplasia. J Obstet Gynaecol Res, 37, 1802-6.

Kantathavorn N, Kietpeerakool C, Suprasert P, et al (2008). Clinical relevance of atypical squamous cells of undetermined significance by the 2001 bethesda system: experience from a cervical cancer high incidence region. Asian Pac J Cancer Prev, 9, 785-8.

Khuakoonratt N, Tangjitgamol S, Manusirivithaya S, et al (2008). Prevalence of high grade squamous intraepithelial lesion (HSIL) and invasive cervical cancer in patients with low grade squamous intraepithelial lesion (LSIL) at cervical pap smear. Asian Pac J Cancer Prev, 9, 253-7.

Limpvanuspong B, Tangjitkamol S, Manusirivithaya S, et al (2008). Prevalence of high grade squamous intraepithelial lesions (HSIL) and invasive cervical cancer in patients with atypical squamous cells of undetermined significance (ASCUS) from cervical pap smears. Southeast Asian J Trop Med Public Health, 39, 737-44.

Massad LS, Einstein MH, Huh WK, et al (2013). 2012 updated consensus guidelines for the management of abnormal cervical cancer screening tests and cancer precursors. J Low Genit Tract Dis, 17, 1-27.

Murillo R, Luna J, Gamboa O, et al (2010). Cervical cancer screening with naked-eye visual inspection in Colombia. Int J Gynaecol Obstet, 109, 230-4.

Nessa A, Nahar KN, Begum SA, et al (2013). Comparison between visual inspection of cervix and cytology based screening procedures in Bangladesh. Asian Pac J Cancer Prev, 14, 7607-11.

Parashari A, Singh V (2013). Reasons for variation in sensitivity and specificity of visual inspection with acetic acid (VIA) for the detection of pre- cancer and cancer lesions of uterine cervix. Asian Pac J Cancer Prev, 14, 7761-2.

Phongsavan K, Phengsavanh A, Wahlstrom R, Marions L (2011). Safety, feasibility, and acceptability of visual inspection with acetic acid and immediate treatment with cryotherapy in rural Laos. Int J Gynaecol Obstet, 114, 268-72.

Rochelson B, Krumholz BA (1983). The "unsatisfactory" colposcopic examination. J Reprod Med, 28, 131-6.

Sankaranarayanan R, Wesley RS (2003). A practical manual on visual screening for cervical neoplasia. Lyon: International Agency for Research on Cancer.

Sankaranarayanan R, Basu P, Wesley RS, et al (2004). Accuracy of visual screening for cervical neoplasia: results from an IARC multicentre study in India and Africa. Int J Cancer, 110, 907-13.

Sankaranarayanan R, Gaffikin L, Jacob M, Sellors J, Robles $S$ (2005). A critical assessment of screening methods for cervical neoplasia. Int J Gynaecol Obstet, 89, 4-12. 
Sankaranarayanan R, Nene BM, Dinshaw KA, et al (2005). A cluster randomized controlled trial of visual, cytology and human papillomavirus screening for cancer of the cervix in rural India. Int J Cancer, 116, 617-23.

Sauvaget C, Fayette JM, Muwonge R, Wesley R, Sankaranarayanan R (2011). Accuracy of visual inspection with acetic acid for cervical cancer screening. Int J Gynaecol Obstet, 113, 14-24.

Solomon D, Davey D, Kurman R, et al (2002). The 2001 Bethesda System: terminology for reporting results of cervical cytology. JAMA, 287, 2114-9.

Vedantham H, Silver MI, Kalpana B, et al (2010). Determinants of VIA (visual inspection of the cervix after acetic acid application) positivity in cervical cancer screening of woman in a peri-urban area in Andhra Pradesh, India. Cancer Epidemiol Biomarkers Prev, 19, 1373-80. 